\title{
The Expected Size of the Rule k Dominating Set
}

\author{
Jennie C. Hansen \\ Actuarial Math and Statistics Department \\ Herriot-Watt University \\ J.Hansen@ma.hw.ac.uk \\ Eric Schmutz \\ Department of Mathematics \\ Drexel University \\ Philadelphia, Pa. 19104 \\ Eric.Jonathan.Schmutz@drexel.edu
}

July 17, 2018

\begin{abstract}
Dai, Li, and Wu proposed Rule k, a localized approximation algorithm that attempts to find a small connected dominating set in a graph. Here we consider the "average case" performance of Rule $k$ for the model of random unit disk graphs constructed from $n$ random points in an $\ell_{n} \times \ell_{n}$ square. If $k \geq 3$ and $\ell_{n}=o(\sqrt{n})$, then the expected size of the Rule $k$ dominating set is $\Theta\left(\ell_{n}^{2}\right)$ as $n \rightarrow \infty$. If $\ell_{n} \leq \sqrt{\frac{n}{10 \log n}}$, then expected size of the minimum CDS is also $\Theta\left(\ell_{n}^{2}\right)$.
\end{abstract}

keywords and phrases: dominating set, localized algorithm, approximation algorithm, performance analysis, probabilistic analysis, Rule $k$, unit disk graph, 


\section{Introduction}

In this paper we consider the problem of finding a small connected dominating set for a unit disk graph $G=(V, E)$, where the vertex set, $V$, is a set of points in $\Re^{2}$. Given the vertex set $V$, the edge set $E$ is determined as follows: an undirected edge $e \in E$ connects vertices $u, v \in V$ (and in this case we say that $u$ and $v$ are adjacent) iff the Euclidean distance between them is less than or equal to one. Unit disk graphs have been used by many authors as simplified mathematical models for the interconnections between hosts in a wireless network, and random unit disk graphs have been used as stochastic models for these networks. e.g. [9], 13], 16], [17, [22, 223. We particularly mention the work of the Hipercom Project, e.g. [18, [19], because it is closely related to our work.

A dominating set in any graph $G=(V, E)$ is a subset $\mathcal{C} \subseteq V$ such that every vertex $v \in V$ either is in the set $\mathcal{C}$, or is adjacent to a vertex in $\mathcal{C}$. We say $\mathcal{C}$ is a connected dominating set if $\mathcal{C}$ is a dominating set and the subgraph induced by $\mathcal{C}$ is connected. Obviously $G$ cannot have a connected dominating set if $G$ itself is not connected. We use the acronym "CDS" for a dominating set $\mathcal{C}$ such that the subgraph induced by $\mathcal{C}$ has the same number of components as $G$ has. In this paper we consider a random unit disk graph model, $\mathcal{G}_{n}$, which is connected with asymptotic probability one. So, in this case, any CDS for $\mathcal{G}_{n}$ will also be connected with high probability.

The identification of a small connected dominating set for the graph which represents the network is an important step in several routing methods. The general idea of CDS-based algorithms is to select a small CDS, and have only those nodes responsible for determining routes [10, 26], 27], 29]. It is believed that, by reducing the number of such nodes, CDS-based algorithms reduce interference between transmitters in the same region and alleviate a related set of problems known collectively as "broadcast storm" 28. Furthermore the cost of finding and maintaining routing information is smaller because fewer nodes are involved. However it is beyond the scope of this paper to consider direct measures of the utility of a small CDS after it has been found. In this paper we consider only a single measure of the algorithms' effectiveness, namely the size of the CDS it finds.

Even with this simple measure of performance, there are non-trivial algorithmic and analytical problems. It is an NP-hard computational problem to find the minimal connected dominating set in a unit disk graph [20. Hence there is considerable practical interest in designing good approximation algorithms for finding small connected dominating sets. See, for example [2], [6], [8], [15], 24], 27]. There have been various efforts to evaluate CDS algorithm's average case performance using simulations. However, with the exception of the theoretical parts of [4, [18, 19], we are not aware of any probabilistic analysis that is proved mathematically.

In this paper we analyze 'Rule $k$ ' $(k \geq 3)$, a family of localized approximation algorithms proposed by $\mathrm{Dai}, \mathrm{Li}$, and $\mathrm{Wu}$ [1], 29]. For each $k$, Rule $k$ attempts to find a small CDS. We first choose an appropriate probability model. Then, in the context of the model, we prove explicit asymptotic bounds on the expected 
size of the dominating set that Rule k produces. Thus our contribution is not the algorithm itself, but rather a mathematically sound analysis of the algorithm.

Before describing Rule $k$, we introduce some notation. We assume that each vertex has a unique identifier taken from a totally ordered set. For convenience, when $|V|=n$, we will use the numbers $1,2, \ldots, n$ as IDs, and will number the vertices accordingly. If $x_{i}$ is any vertex, with ID given by $i$, let $\ldots$ let $N\left(x_{i}\right)$ be the set consisting of $x_{i}$ and any vertices that are adjacent to $x_{i}$. The CDS constructed by the Rule $k$ algorithm is denoted $\mathcal{C}_{k}(V)$, and its cardinality is $C_{k}(V)=\left|\mathcal{C}_{k}(V)\right|$. The elements of $\mathcal{C}_{k}(V)$ are called "gateway nodes". $\mathcal{C}_{k}(V)$ consists of all vertices $x_{i} \in V$ that are not excluded under the following version of Rule k:

Rule k: Vertex $x_{i}$ is excluded from $\mathcal{C}_{k}(V)$ iff $N\left(x_{i}\right)$ contains at least one set of $k$ vertices $x_{i_{1}}, x_{i_{2}}, \ldots x_{i_{k}}$ such that

- $i_{1}>i_{2}>\cdots>i_{k}>i$, and

- The subgraph induced by $\left\{x_{i_{1}}, x_{i_{2}}, \cdots, x_{i_{k}}\right\}$ is connected, and

- $N\left(x_{i}\right) \subseteq \bigcup_{t=1}^{k} N\left(x_{i_{t}}\right)$.

$\mathrm{Wu} \mathrm{Li}$ and Dai proved that $\mathcal{C}_{k}(V)$ is a CDS, and they conjectured that the Rule $k$ dominating set is, in some sense, small on average. The main result in this paper is a proof of their conjecture.

The rest of this paper is organized as follows. In the next section we specify the model and define the random unit disk graph, $\mathcal{G}_{n}$. In Sections 3 we prove a local coverage theorem that is needed in section 4 to prove an upper bound for $E\left(C_{k}(V)\right)$ Finally, in the remainder of the paper, we discuss lower bounds and optimality issues. The appendix deals with a related algorithm called the Marking Process.

\section{Choice of Models}

Before estimating the expected size of the Rule $k$ dominating set, we must specify the underlying probability model. For any real number $\ell>1$, let $\mathcal{Q}(\ell)$ be an $\ell \times \ell$ square in $\Re^{2}$. The particular choice of a square will be immaterial, but its size will be very important. Let $\Omega_{n, \ell}=\mathcal{Q}(\ell) \times \mathcal{Q}(\ell) \times \ldots \times \mathcal{Q}(\ell)$ be the $n$-fold product space with the usual product topology. For each $n \geq 1$, let $X_{n, \ell, 1}, X_{n, \ell, 2}, \ldots, X_{n, \ell, n}$ be a sequence of random points selected independently from a uniform distribution on $\mathcal{Q}(\ell)$ and let $\mathbf{P}_{n, \ell}$ denote the uniform probability measure on $\Omega_{n, \ell}$ induced by the random variables $X_{n, \ell, 1}, X_{n, \ell, 2}, \ldots, X_{n, \ell, n}$. Finally, let $\mathcal{G}(n, \ell)$ be the random unit disk graph with vertex set $V_{n, \ell}=$ $\left\{X_{n, \ell, 1}, X_{n, \ell, 2}, \ldots, X_{n, \ell, n}\right\}$ that is formed from these vertices by putting an edge between two vertices iff the Euclidean distance between the two vertices is less than or equal to one. 
We want to estimate the "average" size of $\mathcal{C}_{k}\left(V_{n, \ell}\right)$ for large networks. As it stands, the expected value $E_{n, \ell}\left(C_{k}\right)\left[=E\left(C_{k}\left(V_{n, \ell}\right)\right)\right]$ is defined with respect to the probability measure $\mathbf{P}_{n, \ell}$ on $\Omega_{n, \ell}$ and depends on both $n$ and $\ell$. We shall not however attempt any multivariate asymptotic estimates. Instead, we choose a suitable sequence, $\left\langle\ell_{n}\right\rangle_{n=1}^{\infty}$, and consider the expected value $E_{n, \ell_{n}}\left(C_{k}\right)$ with respect to $\mathbf{P}_{n, \ell_{n}}$ as $n \rightarrow \infty$. To simplify notation throughout, we will (usually) suppress the dependence on the choice of a sequence $\left\langle\ell_{n}\right\rangle_{n=1}^{\infty}$. Thus we write $\mathcal{G}_{n}$ instead of $\mathcal{G}\left(n, \ell_{n}\right)$, and write $E_{n}\left(C_{k}\right)$ instead of $E_{n, \ell_{n}}\left(C_{k}\right)$. Suppressing even $n$, we write $\mathcal{Q}$ instead of $\mathcal{Q}\left(\ell_{n}\right)$, and $\mathbf{P}$ instead of $\mathbf{P}_{n, \ell_{n}}$.

Conditions on the growth rate of $\ell_{n}$ will be clear from the statements of theorems. However, to provide some perspective on our choice of growth rates for $\ell_{n}$, we mention that it is known that the threshold for connectivity is $\ell_{n}=$ $\Theta(\sqrt{n / \log n})$; if $\ell_{n}$ grows faster than this, then the random unit disk graph $\mathcal{G}_{n}$ will be disconnected with probability $1-o(1)$ as $n \rightarrow \infty$. In this case, with high probability, $\mathcal{C}_{k}\left(V_{n, \ell}\right)$ will not be a connected dominating set for $\mathcal{G}_{n}$. More precise versions of these remarks are provided in the new book by Penrose 25] which gives an up to date survey of random geometric graphs.

Finally, throughout the remainder of this paper we adopt the following notation. For any points $p$ and $q$ in $\Re^{2}$, let $d(p, q)$ denote the ordinary Euclidean distance between $p$ and $q$ in $\Re^{2}$.

\section{Local Coverage by $k$ vertices}

The next lemma is a purely geometric result which we require for the proof of Theorem 2. To state the lemma, we need some notation. Let $\delta=\frac{1}{2}-\frac{\sqrt{3}}{4}=$ $.0669 \ldots$, and define $\rho$ by $\rho+2 \delta=1 ; \rho=\frac{\sqrt{3}}{2}=.866 \ldots$ Let $p$ be any point in $\mathcal{Q}$, and let $D^{\prime}=D_{1}(p) \cap \mathcal{Q}$ be the set of points in the square $\mathcal{Q}$ whose distance from $p$ is one or less.

Lemma 1 There exist points $z_{0}, z_{1}, z_{2} \in D^{\prime}$ such that the following two conditions are satisfied:

- for $s=0$ and $s=1, d\left(z_{s}, z_{s+1}\right) \leq 1-2 \delta$

- $D^{\prime} \subseteq \bigcup_{s=0}^{2} D_{\rho}\left(z_{s}\right)$.

Proof: Consider first the case where $D_{1}(p) \subseteq \mathcal{Q}$, i.e. $p$ is a point that is not near the boundary of the square. We may, without loss of generality, choose the coordinate system such that $p=(0,0)$ and such that the axes are parallel to the sides of the square $\mathcal{Q}$. For $s=0,1,2$, let $S_{s}$ be the sector of $D_{1}(p)$ consisting of those points whose polar coordinates $(r, \theta)$ satisfy $r \leq 1$ and $\frac{(2 s-1) \pi}{3} \leq \theta \leq$ $\frac{(2 s+1) \pi}{3}$. Let $z_{s}$ be the point in $S_{s}$ whose polar coordinates are $\left(\frac{1}{2}, \frac{2 \pi s}{3}\right)$. Then the first condition is satisfied: $d\left(z_{s}, z_{s+1}\right)=\sin \frac{\pi}{3}=1-2 \delta$. It is also straightforward to check that for $s=0,1,2, S_{s} \subseteq D_{\rho}\left(z_{s}\right)$ and so the second condition is satisfied. 
Now consider the remaining case where $D_{1}(p)$ meets the boundary of $\mathcal{Q}$. Choose points $z_{0}, z_{1}, z_{2}$ as before so that $D_{1}(p) \subseteq \bigcup_{s=0}^{2} D_{\rho}\left(z_{s}\right)$ and $d\left(z_{s}, z_{s+1}\right) \leq$ $1-2 \delta$. We are not done because one or more of the points $z_{s}$ may not lie in $\mathcal{Q}$. In particular, if $z_{s} \notin \mathcal{Q}$, then there is a (unique) $z_{s}^{\prime} \in \mathcal{Q}$ such that $d\left(z_{s}, z_{s}^{\prime}\right)=$ $\inf \left\{d\left(z_{s}, z\right): z \in \mathcal{Q}\right\}$. We replace $z_{s}$ by $z_{s}^{\prime}$ and observe that every point of $D^{\prime}$ is closer to $z_{s}^{\prime}$ than it is to the original point $z_{s}$. Hence $S_{s} \cap \mathcal{Q} \subseteq D_{\rho}\left(z_{s}^{\prime}\right)$. After replacing all $z_{s}$ such that $z_{s} \notin \mathcal{Q}$ by the corresponding $z_{s}^{\prime}$ we obtain three points that satisfy the conditions of the lemma.

Fix $k \geq 3$, the $k$ in "Rule k". Suppose $m$ points $P_{1}, P_{2}, \ldots, P_{m}$ are selected independently and uniform randomly in $D^{\prime}(p)$. Let $\mathcal{K}_{m}$ be the event that, for some $1 \leq i_{0}<i_{1}<i_{2}<\ldots<i_{k-1} \leq m$, we have:

- $D^{\prime} \subseteq \bigcup_{s=0}^{k-1} D_{1}\left(P_{i_{s}}\right)$, and

- the unit disk graph with vertices $P_{i_{0}}, P_{i_{1}}, \ldots, P_{i_{k-1}}$ is connected.

We note that event $\mathcal{K}_{m}$ implies that the random unit disk graph which is formed from the vertices $P_{1}, P_{2}, \ldots, P_{m}$ has a $k$-point connected dominatng set. With this notation we can state

Theorem 2 There is a positive constant $\alpha<1$ and a positive constant $m_{k}$ such that, for all $m>m_{k}, \operatorname{Pr}\left(\mathcal{K}_{m}\right)>1-4 \alpha^{m}$.

Proof: Choose points $z_{0}, z_{1}, z_{2}$ as in the proof of Lemma 1 If $z$ is any point in $D_{\delta}\left(z_{s}\right)$, then for all $y \in S_{s}, d(z, y) \leq d\left(z, z_{s}\right)+d\left(z_{s}, y\right) \leq \delta+\rho<1$. Let $\mathcal{E}_{s}$ be the event that none of the $m$ random points $P_{1}, P_{2}, \ldots, P_{m}$ lies in $D_{\delta}\left(z_{s}\right)$. Then

$$
\operatorname{Pr}\left(\mathcal{E}_{s}\right)=\left(1-\frac{\operatorname{Area}\left(D_{\delta}\left(z_{s}\right) \bigcap \mathcal{Q}\right)}{\operatorname{Area}\left(D^{\prime}\right)}\right)^{m} .
$$

Note that $\operatorname{Area}\left(D_{\delta}\left(z_{s}\right) \cap \mathcal{Q}\right) \geq \frac{1}{4} \operatorname{Area}\left(D_{\delta}\left(z_{s}\right)\right)=\frac{\pi \delta^{2}}{4}$, and that $\operatorname{Area}\left(D^{\prime}\right) \leq$ $\operatorname{Area}\left(D_{1}(p)\right)=\pi$. If we let $\alpha=1-\frac{\delta^{2}}{4}=.998 \ldots$, then $\alpha<1$, and for $s=0,1,2$,

$$
\operatorname{Pr}\left(\mathcal{E}_{s}\right) \leq \alpha^{m} .
$$

It follows from(2) that $\operatorname{Pr}\left(\mathcal{K}_{m}\right) \geq 1-3 \alpha^{m}$ since $\mathcal{E}_{0}^{c} \cap \mathcal{E}_{1}^{c} \cap \mathcal{E}_{2}^{c} \subseteq \mathcal{K}_{m}$, and the proof is complete if $k=3$.

Now suppose that $k>3$, and let $Y$ be the number of the $m$ random points that lie in $D_{\rho}\left(z_{2}\right) \cap \mathcal{Q}$. Since $\{Y \geq k\} \cap\left(\bigcap_{s=0}^{2} \mathcal{E}_{s}^{c}\right) \subseteq \mathcal{K}_{m}$, we have

$$
\operatorname{Pr}\left(\mathcal{K}_{m}\right) \geq 1-\operatorname{Pr}\left(\bigcup_{s=0}^{2} \mathcal{E}_{s}\right)-\operatorname{Pr}(Y<k) .
$$

But $Y$ has a binomial $(m, \tilde{p})$ distribution, where

$$
\tilde{p}=\frac{\operatorname{Area}\left(D_{\rho}\left(z_{s}\right) \cap \mathcal{Q}\right)}{\operatorname{Area}\left(D^{\prime}\right)}>\frac{\pi \rho^{2} / 4}{\pi}=\frac{3}{16} .
$$


Hence, for all $m \geq k$,

$$
\begin{aligned}
& \operatorname{Pr}(Y<k)=\sum_{j=0}^{k-1}\left(\begin{array}{c}
m \\
j
\end{array}\right) \tilde{p}^{j}(1-\tilde{p})^{m-j} \\
& <m^{k}(1-\tilde{p})^{m-k}<\left(\frac{16 m}{13}\right)^{k} \cdot\left(\frac{13}{16}\right)^{m} .
\end{aligned}
$$

Since $\frac{13}{16}<\alpha$, it follows that,as $m \rightarrow \infty$,

$$
\operatorname{Pr}(Y<k)=o\left(\alpha^{m}\right) .
$$

Put (2), (3), and (7) together to conclude: there is a positive constant $m_{k}$ such that, for all $m>m_{k}$,

$$
\operatorname{Pr}\left(\mathcal{K}_{m}^{c}\right)<4 \alpha^{m}
$$

\section{Analysis of Rule $\mathrm{k}$}

In this section, we assume that $\ell_{n}=o(\sqrt{n})$ as $n \rightarrow \infty$. Also, in this section, let $U_{k}=\sum_{i=1}^{n} I_{i}$ be a sum of indicator variables where $I_{i}=1$ iff node $i$ is not included in $\mathcal{C}_{k}(V)$ under Rule k. Thus Rule $k$ selects a dominating set $\mathcal{C}_{k}(V)$ having $C_{k}(V)=n-U_{k}$ vertices, and it is desirable for $U_{k}$ to be large. Our goal in this section is to prove that, for all $k>2, E\left(U_{k}\right) \geq n-O\left(\ell_{n}^{2}\right)$.

Let $\lambda_{n}=n-\ell_{n}^{2}$, and let let $X_{1}, X_{2}, \ldots, X_{n}$ be independent, uniformly distributed random points in $\mathcal{Q}$, namely the locations of vertices. (Here we are again simplifying notation by writing $X_{i}$ instead of $X_{n, \ell_{n}, i}$.) Let $\rho_{i}$ be the number of neighbors of vertex $i$ having a larger ID, i.e. the number of $j>i$ such that $d\left(X_{i}, X_{j}\right) \leq 1$.

Lemma 3 If $\ell_{n}=o(\sqrt{n})$, then

$$
\mathbf{P}\left(\rho_{i}<\frac{(n-i) \pi}{8 \ell_{n}^{2}}\right) \leq \exp \left(\frac{-(n-i) \pi}{32 \ell_{n}^{2}}\right) .
$$

Proof: Let $\left|D_{1}\left(X_{i}\right)\right|=\operatorname{Area}\left(D_{1}\left(X_{i}\right) \cap \mathcal{Q}\right)$ be the area of the set of points in $\mathcal{Q}$ whose distance from $X_{i}$ is one or less. Thus $\left|D_{1}\left(X_{i}\right)\right|=\pi$ unless $X_{i}$ happens to fall near the border, and in all cases $\left|D_{1}\left(X_{i}\right)\right| \geq \frac{\pi}{4}$. Given $\left|D_{1}\left(X_{i}\right)\right|$, the variable $\rho_{i}$ has a Binomial $\left(n-i, \frac{\left|D_{1}\left(X_{i}\right)\right|}{\ell_{n}^{2}}\right)$ distribution. Therefore Chernoff's bound on the lower tail distribution gives

$$
\begin{gathered}
\mathbf{P}\left(\rho_{i}<\frac{(n-i) \pi}{8 \ell_{n}^{2}}|| D_{1}\left(X_{i}\right) \mid\right)= \\
\mathbf{P}\left(\rho_{i}<\frac{\pi}{8\left|D_{1}\left(X_{i}\right)\right|} \cdot \frac{\left|D_{1}\left(X_{i}\right)\right|(n-i)}{\ell_{n}^{2}}|| D_{1}\left(X_{i}\right) \mid\right)
\end{gathered}
$$




$$
\begin{gathered}
\leq \exp \left(-\left(1-\frac{\pi}{8\left|D_{1}\left(X_{i}\right)\right|}\right)^{2} \cdot \frac{\left|D_{1}\left(X_{i}\right)\right|(n-i)}{2 \ell_{n}^{2}}\right) \\
\leq \exp \left(\frac{-(n-i) \pi}{32 \ell_{n}^{2}}\right) .
\end{gathered}
$$

Theorem 4 If $k>2$, then $E_{n}\left(C_{k}\right)=O\left(\ell_{n}^{2}\right)$.

Proof: Let $\mathcal{B}_{i}$ be the event that $\rho_{i} \geq \frac{(n-i) \pi}{8 \ell_{n}^{2}}$. By Lemma 3

$$
\mathbf{P}\left(I_{i}=1\right) \geq \mathbf{P}\left(I_{i}=1 \mid \mathcal{B}_{i}\right) \mathbf{P}\left(\mathcal{B}_{i}\right) \geq \mathbf{P}\left(I_{i}=1 \mid \mathcal{B}_{i}\right)\left(1-\exp \left(\frac{-(n-i) \pi}{32 \ell_{n}^{2}}\right)\right)
$$

Now suppose that $i \leq \lambda_{n}=n-\ell_{n}^{2}$, and observe that

$$
\mathbf{P}\left(I_{i}=1 \mid \mathcal{B}_{i}\right)=\sum_{v \geq \frac{(n-i) \pi}{8 \ell_{n}^{2}}} \mathbf{P}\left(I_{i}=1 \mid \rho_{i}=v\right) \mathbf{P}\left(\rho_{i}=v \mid \mathcal{B}_{i}\right) .
$$

To estimate this, observe that

$$
\mathbf{P}\left(I_{i}=1 \mid \rho_{i}=v\right)=\int_{\mathcal{Q}} \mathbf{P}\left(I_{i}=1 \mid \rho_{i}=v, X_{i}=\vec{x}\right) f_{X_{i}}\left(\vec{x} \mid \rho_{i}=v\right) d \vec{x}
$$

where $f_{X_{i}}\left(\vec{x} \mid \rho_{i}=v\right)$ is the conditional density of $X_{i}$ on the square $\mathcal{Q}$ given that $\rho_{i}=v$. For $v>(n-i) \pi / 8 \ell_{n}^{2}$, Theorem 2 yields

$$
\mathbf{P}\left(I_{i}=1 \mid \rho_{i}=v, X_{i}=x\right) \geq 1-4 \alpha^{v} \geq 1-4 \alpha^{(n-i) \pi / 8 \ell_{n}^{2}} .
$$

Putting this back into (11) and then (10), we get

$$
\mathbf{P}\left(I_{i}=1 \mid \mathcal{B}_{i}\right) \geq 1-4 \alpha^{(n-i) \pi / 8 \ell_{n}^{2}},
$$

and therefore

$$
\begin{aligned}
\mathbf{P}\left(I_{i}=1\right) \geq \mathbf{P}\left(I_{i}=\right. & \left.1 \mid \mathcal{B}_{i}\right) \mathbf{P}\left(\mathcal{B}_{i}\right) \geq\left(1-4 \alpha^{(n-i) \pi / 8 \ell_{n}^{2}}\right)\left(1-\exp \left(-\frac{(n-i) \pi}{32 \ell_{n}^{2}}\right)\right) \\
& \geq 1-4 \alpha^{(n-i) \pi / 8 \ell_{n}^{2}}-\exp \left(-\frac{(n-i) \pi}{32 \ell_{n}^{2}}\right) .
\end{aligned}
$$

Recall that $\lambda_{n}=n-\ell_{n}^{2}$, and that the foregoing estimates were valid for all $i \leq \lambda_{n}$. Putting $j=n-i$, we get

$$
\begin{gathered}
E\left(U_{k}\right) \geq \sum_{i=1}^{\lambda_{n}} \mathbf{P}\left(I_{i}=1\right)=\sum_{i=1}^{\lambda_{n}}\left(1-4 \alpha^{(n-i) \pi / 8 \ell_{n}^{2}}-\exp \left(-\frac{(n-i) \pi}{32 \ell_{n}^{2}}\right)\right) \\
\geq \lambda_{n}-4 \sum_{j \geq \ell_{n}^{2}}\left(\alpha^{\pi / 8 \ell_{n}^{2}}\right)^{j}-\sum_{j \geq \ell_{n}^{2}}\left(\exp \left(-\pi / 32 \ell_{n}^{2}\right)\right)^{j} \\
=n-O\left(\ell_{n}^{2}\right) .
\end{gathered}
$$




\section{Lower Bound}

If a vertex $v$ has higher ID than any of its neighbors, then it cannot be eliminated under Rule $k$. This simple observation is the basis for

Theorem 5 If $\ell_{n}=o(\sqrt{n})$, then, for all sufficiently large $n$, the expected size of the Rule $k$ dominating set is more than $\ell_{n}^{2} / 4$.

Proof: Let $L_{k}=\sum_{i=1}^{n} I_{i}$, where $I_{i}=1$ iff node $i$ has a higher ID that all the nodes in $D_{1}\left(X_{i}\right)$. Note that $I_{i}=1$ iff the nodes $X_{i+1}, X_{i+2}, \ldots, X_{n}$ all fall outside the disk $D_{1}\left(X_{i}\right)$. Therefore

$$
\mathbf{P}\left(I_{i}=1\right)=\left(1-\frac{\left|D_{1}\left(X_{i}\right)\right|}{\ell_{n}^{2}}\right)^{n-i} \geq\left(1-\frac{\pi}{\ell_{n}^{2}}\right)^{n-i}
$$

Therefore

$$
E\left(L_{n}\right) \geq \sum_{i=1}^{n}\left(1-\frac{\pi}{\ell_{n}^{2}}\right)^{n-i}=\frac{\ell_{n}^{2}}{\pi}\left(1-\left(1-\frac{\pi}{\ell_{n}^{2}}\right)^{n}\right)=\frac{\ell_{n}^{2}}{\pi}(1-o(1)) .
$$

\section{Optimality}

For this section, $\ell_{n} \leq \sqrt{\frac{n}{a \log n}}$, where $a$ is a constant greater than 9. It is easy to verify that, with asymptotic probability one, there exists a CDS, $C_{\text {rand }}$, having $O\left(\ell_{n}^{2}\right)$ vertices: simply partition the square $\mathcal{Q}$ into $\left\lfloor 3 \ell_{n}\right\rfloor^{2}$ equal-sized squares, each with sides of length $s_{n}=\frac{\ell_{n}}{\left[3 \ell_{n}\right\rfloor}=\frac{1}{3}+O\left(\frac{1}{\ell_{n}}\right)$, and then pick one node from each of these small squares. More explicitly, for $0 \leq i, j<\left\lfloor 3 \ell_{n}\right\rfloor$, let $Q_{i, j}=\left\{(x, y): i s_{n} \leq x<(i+1) s_{n}\right.$ and $\left.j s_{n} \leq x<(j+1) s_{n}\right\}$. Let $\mathcal{B}$ be the event that each of the $\left\lfloor 3 \ell_{n}\right\rfloor^{2}$ small squares contains one or more nodes. By Boole's inequality,

$$
\begin{array}{rl}
\mathbf{P}\left(\mathcal{B}^{c}\right) \leq 9 \ell_{n}^{2} & \mathbf{P}\left(Q_{1,1} \text { is empty }\right)=9 \ell_{n}^{2}\left(1-\frac{1}{\left\lfloor 3 \ell_{n}\right\rfloor^{2}}\right)^{n} \\
= & 9 \ell_{n}^{2} \exp \left(-\frac{n}{9 \ell_{n}^{2}}\left(1+O\left(\frac{1}{\ell_{n}^{2}}\right)\right)\right. \\
& <\frac{n}{\log n} e^{-\log n}=O\left(\frac{1}{\log n}\right) .
\end{array}
$$

Now given the vertices $V=\left\{X_{1}, X_{2}, \ldots, X_{n}\right\}$, we construct $C_{\text {rand }} \subseteq V$ as follows: For each $1 \leq i, j \leq\left\lfloor 3 \ell_{n}\right\rfloor$, if $Q_{i, j}$ contains at least one vertex, then select one vertex $V_{i, j}$ uniform randomly from among the vetices in $Q_{i, j}$, and include $V_{i, j}$ in $C_{\text {rand }}$. Thus $C_{\text {rand }}$ is a (random) set of at most $\left\lfloor 3 \ell_{n}\right\rfloor^{2}$ nodes. It can contain 
fewer nodes (possibly as few as one), but with asymptotic probability $1, C_{\text {rand }}$ contains exactly $\left\lfloor 3 \ell_{n}\right\rfloor^{2}$ vetices and is a CDS.

It is worth pointing out that this existence argument cannot be used in a straight-forward way as the basis for a localized algorithm because the nodes do not know their own locations in the network. One of the main advantages of the Rule $\mathrm{k}$ algorithm is that a vertex makes its decision based on very limited information, namely its list of neighbors and their lists of neighbors.

Nevertheless, the existence argument is useful for us because it leads to a lower bound the size that a CDS can have. The following argument was influenced by [21]. The appendix of [11] is also pertinent, but we do not see how to turn the discussion there into a mathematically rigorous proof.

Theorem 6 below is based on from the following observation: If $v$ is any point in $\mathcal{Q}$, then at most 81 nodes of $C_{\text {rand }}$ are in $D_{1}(v)$. In particular, if $C_{o p t}$ is a minimum sized CDS, and $v$ is a node in $C_{o p t}$, then $N(v)$ includes at most 81 nodes of $C_{\text {rand }}$. But $C_{o p t}$ is a dominating set; therefore every node in $C_{\text {rand }}$ must be in $N(v)$ for at least one $v \in C_{o p t}$. We therefore have a lower bound of the size of $C_{\text {opt }}$ :

$$
\left|C_{\text {opt }}\right| \geq \frac{1}{81}\left|C_{\text {rand }}\right|
$$

Combining (24) with (23), we get

Theorem 6 Suppose $a>9$, and $\ell_{n} \leq \sqrt{\frac{n}{a \log n}}$ for all $n$. Then there is $a$ constant $B>0$ such that, for all $n>1$,

$$
\mathbf{P}_{n, \ell_{n}}\left(\left|C_{o p t}\right|<\frac{1}{10} \ell_{n}^{2}\right)<\frac{B}{\log n} .
$$

Corollary $7 \quad E\left(\left|C_{\text {opt }}\right|\right)=\Theta\left(\ell_{n}^{2}\right)$

Proof: From (24), we have

$$
\begin{gathered}
E\left(\left|C_{\text {opt }}\right|\right) \geq \frac{1}{81} E\left(\left|C_{\text {rand }}\right|\right) \\
\geq \frac{1}{81} \mathbf{P}\left(\left|C_{\text {rand }}\right|=\left\lfloor 3 \ell_{n}^{2}\right\rfloor\right) \cdot\left\lfloor 3 \ell_{n}^{2}\right\rfloor \\
=\frac{1}{81}\left(1-O\left(\frac{1}{\log n}\right)\right)\left\lfloor 3 \ell_{n}^{2}\right\rfloor=\Theta\left(\ell_{n}^{2}\right) .
\end{gathered}
$$

\section{Discussion}

In this paper we have analyzed Rule $k$ only for $k>2$. For $k<3$, the analysis is different and quite a bit more complicated. The analysis for that case is treated in a subsequent paper. Also, here we have only analyzed the application of Rule 
$k$ on the entire vertex set of $\mathcal{G}_{n}$. Clearly Rule $k$ could also be used in conjunction with other heuristics in order to construct a "small" CDS. For example, Wu and Li have proposed the "Marking Process", an algorithm for selecting an initial CDS $\mathcal{M}$. They recommended that the Marking Process be followed by Rules 1 and 2. Dai and Wu subsequently proposed the more general Rule $k$. The various Rules $1,2,3, \ldots$ can be applied one after the other up to some largest $k$. Dai Li and $\mathrm{Wu}$ mark the nodes in the CDS, and with each new rule application, the set of marked nodes shrinks.

In the case where $\ell_{n} \rightarrow \infty$ and $\ell_{n}<\sqrt{n / 3 \log n}$, it can be shown (see Appendix 1) that asymptotically nothing is gained by applying the Marking Process before applying Rule $k$. It may be possible to obtain further reductions in the size of the heuristic CDS by successive applications of Rules $1,2, . ., k$ as proposed by Dai $\mathrm{Li}$ and $\mathrm{Wu}$. However, the rigorous analysis of the $\mathrm{Dai} \mathrm{Li}$ and $\mathrm{Wu}$ heuristic is complicated due to dependence between the variables at the various stages in the analysis of the heuristic. For example, it seems much harder to estimate $E\left(\left|\mathcal{C}_{k+1}\left(\mathcal{C}_{k}(V)\right)\right|\right)$ than it is to estimate $E\left(\left|\mathcal{C}_{k+1}(V)\right|\right)$ (say). Our analysis only considered $E\left(\left|\mathcal{C}_{k}(V)\right|\right)$ for any fixed $k>2$ and we have shown that in this case the average size of $\mathcal{C}_{k}(V)$ is of the same order as the size of the optimal CDS. So, even a simple application of Rule $k$ to the entire vertex set $V$ produces, on average, a "good" CDS. 
Acknowledgement We thank Li Sheng and Harish Sethu helpful comments.

\section{References}

[1] C.Adjih,P.Jacquet, L.Viennot,Computing Connected Dominating Sets with Multipoint Relays, INRIA RR-4597 (2002).

[2] K.M.Alzoubi,P.J.Wan,and O.Frieder, Distributed Heuristics for Connected Dominating Sets in Wireless Ad Hoc Networks, Journal of Communications and Networks,4 (1) (2002) 1-8.

[3] M.J.B.Appel and R.P.Russo, The minimum vertex degree of a graph on uniform points in $[0,1]^{d}$, Advances in Appl.Probab 29 (3) (1997) 582-594.

[4] E.Baccelli and P.Jacquet, Flooding Techniques in Mobile Ad Hoc Networks, INRIA RR-5002 (2003).

[5] T.Camp and B.Williams, Comparison of Broadcasting Techniques for Mobile Ad Hoc Networks, Proceedings of the 3'rd ACM International Symposium on Mobile Ad Hoc Networking and Computing, MobiHoc 2002 (2002) 194205.

[6] M.Cardei,X.Cheng,X.Cheng, D.Du,Connected Domination in Multihop Ad Hoc Wireless Networks, JCIS (2002) 251-255.

[7] G.B.Chae, E.M. Palmer, and W.C. Siu, Geodetic Number of Random Graphs of Diameter Two , Australasian Journal of Combinatorics 26 (2002) 11-20.

[8] B. Chen, K. Jamieson, H. Balakrishnan, R. Morris , Span: An EnergyEfficient Coordination Algorithm for Topology Maintenance in Ad Hoc Wireless Networks Proc. of the 6th ACM MOBICOM Conf., Rome, Italy, July 2001.

[9] B.N. Clark,C.J. Colburn, and D.J.Johnson, Unit Disk Graphs,Discrete Mathematics 86(1-3) (1990) 165-177.

[10] B.Das and V. Bharghavan Routing in Ad-Hoc Networks Using Minimum Connected Dominating Sets, International Conference on Communications 1 (1997) 376-380.

[11] F.Dai and J.Wu, An Extended Localized Algorithm for Connected Dominating Set Formation in Ad Hoc Wireless Networks, IEEE Transactions on Parallel and Distributed Systems, 15 (10) 2004.

[12] F.Dai and J.Wu, Performance analysis of broadcast protocols in ad hoc networks based on self pruning, IEEE Trans.on Parallel and Distributed Systems 15 (11) (2004).

[13] E.N.Gilbert, Random Plane Networks, J.Soc.Indust.Appl.Math. 9 (1961) 533.

[14] A.Godbole and B.Wielund, On the Domination Number of a Random Graph, Electronic Journal of Combinatorics 8 \#R37 (2001) 
[15] S.Guha and S.Khuller, Approximation algorithms for connected dominating sets, Algorithmica 20 (4) (1998) 374-387.

[16] P.Gupta and P.R.Kumar, "Critical power for asymptotic connectivity in wireless networks", in Stochastic Analysis, Control, Optimization and Applications, Birkhauser (1999) 547-566.

[17] W.K.Hale, Frequency Assignment: Theory and Applications,Proc. IEEE 68 (1980) 1497-1514.

[18] P.Jacquet, Analytical Results on Connected Dominating Sets in Mobile Ad Hoc Networks, INRIA RR-5173 (2004).

[19] P.Jacquet, A.Laouiti,P.Minet,L.Viennot, Performance of Mutltipoint Relaying in Ad Hoc Mobil Routing Protocols, In "Networking 2002" Lecture Notes in Computer Science 2345 (2002) 387-398.

[20] D.Lichtenstein, Planar formulae and their uses, SIAM J.Comput. 11(2) (1982) 329-343.

[21] M.V.Marathe,H.Breu,H.B.Hunt,S.S.Ravi,and D.J.Rosenkrantz, Simple Heuristics for Unit Disk Graphs, Networks 25 no.2 (1995) 59-68.

[22] C.McDiarmid, Discrete mathematics and radio channel assignment, Recent Advances in algorithms and combinatorics, 27-63, CMS Books Math (2003).

[23] C.McDiarmid, Random channel assignment in the plane, Random Structures and Algorithms 22 (2) 187-212.

[24] W.Peng and X.Lu,On the reduction of broadcast redundancy in mobile ad hoc networks, Proceedings of the 1'st ACM International Symposium on Mobile Ad Hoc Networking (2000)129-130.

[25] Random Geometric Graphs, Oxford Studies in Probability 5, Oxford University Press, (2003) ISBN 0-19-850626-0.

[26] R.Sivakumar, B.Das, and V. Bharghavan, , Spine-based routing in ad hoc networks, Cluster Computing 1 (2) (1998) 237-248.

[27] I. Stojmenovic, M. Seddigh, J. Zunic, Dominating sets and neighbor elimination based broadcasting algorithms in wireless networks, IEEE Transactions on Parallel and Distributed Systems, Vol. 13, No. 1,(2002), 14-25.

[28] Y.C.Tseng,S.Y.Ni, Y.S.Chen, J.P.Sheu,The broadcast storm problem in a mobile ad hoc network, Wireless Networks 8(2-3) (2002) 153-167.

[29] J. Wu and H.Li, On calculating connected dominating set for efficient routing in ad hoc wireless networks, Workshop on Discrete Algorithms and Methods for MOBILE Computing and Communications (1999) 7-14.

[30] A.C.C.Yao, On constructing spanning trees in $k$ dimensional spaces and related problems, SIAM J.Computing 11 (4) (1982) 721-736. 


\section{Appendix 1: The Marking Process}

Wu and Li 29] proposed the following method for selecting an initial CDS $\mathcal{M}$ :

Marking Process: a node is included in $\mathcal{M}$ iff it has two neighbors that are not adjacent (i.e. not directly connected by and edge).

Suppose we apply the Marking Process to the random graph $\mathcal{G}_{n}$. Let $M=|\mathcal{M}|$ be the number of vertices marked by the marking process. In this appendix, let $I_{i}=1$ iff the $i$ th vertex gets marked, i.e. vertex $i$ has two non-adjacent neighbors. Let $I_{i}=0$ otherwise. Thus $M=\sum_{i=1}^{n} I_{i}$ is the number of marked vertices. Our goal is to establish the following asymptotic estimate for the expected value of $M$.

Theorem $8 E(M)=n-O\left(n \exp \left(-\frac{n}{\ell_{n}^{2}}\right)\right)$.

Proof: Since the $I_{i}$ 's are identically distributed, we have

$$
E(M)=n \mathbf{P}\left(I_{1}=1\right) .
$$

It therefore suffices to prove that $\mathbf{P}\left(I_{1}=0\right)=O\left(\exp \left(-\frac{n}{\ell_{n}^{2}}\right)\right)$. For any $i$, and any $r>0$, let $D_{r}(i)$ be the disk of radius $r$ centered at the vertex labelled $i$. If vertex 1 happens to fall near the boundary of $\mathcal{Q}$, then it may happen that part of $D_{1}(1)$ is not entirely contained in $\mathcal{Q}$. But in any case we can partition $D_{1}(1)$ into four quarter disks and select one of the four quarter disks $K$ in such a way $K$ is contained in $\mathcal{Q}$. If $\varphi$ is the axis of symmetry of $K$, let $B_{1}$ be the set of points in $K$ whose distance from $\varphi$ is greater than $\frac{1}{2}$. Note that $B_{1}$ consists of two disjoint components $B_{1}^{+}, B_{1}^{-}$, and that the distance from $B_{1}^{+}$to $B_{1}^{-}$is 1 . Hence vertex 1 will be marked if both $B_{1}^{+}$and $B_{1}^{-}$contain at least one of the other $n-1$ vertices. Define $\mathcal{B}_{1}$ to be the event that both $B_{1}^{+}$and $B_{1}^{-}$contain at least one of the other $n-1$ vertices. In this section only, define $\alpha$ to be the area of $B_{1}^{+}$. The probability that $B_{1}^{+}$contains none of the other $n-1$ nodes is $\left(\frac{\ell_{n}^{2}-\alpha}{\ell_{n}^{2}}\right)^{n-1}$. The same is true of $B_{1}^{-}$. Hence

$$
\mathbf{P}\left(I_{1}=0\right) \leq 2\left(\frac{\ell_{n}^{2}-\alpha}{\ell_{n}^{2}}\right)^{n-1}=O\left(e^{-n / \ell_{n}^{2}}\right) .
$$

Corollary $9 \mathbf{P}(M \neq n)=O\left(n e^{-n / \ell_{n}^{2}}\right)$.

Proof: By Boole's inequality,

$\mathbf{P}(M \neq n)=\mathbf{P}\left(I_{i}=0\right.$ for some $\left.i\right) \leq n \mathbf{P}\left(I_{1}=0\right)=O\left(n e^{-n / \ell_{n}^{2}}\right)$.

Now fix $k \geq 2$, and let $C_{k}=\left|\mathcal{C}_{k}(V)\right|$ be the number of vertices in the CDS which is constructed when Rule $\mathrm{k}$ is applied to all nodes in the network. Let $C_{k}^{\prime}=\left|\mathcal{C}_{k}(\mathcal{M})\right|$ be the number of vertices in the CDS which is constructed when Rule $\mathrm{k}$ is applied to $\mathcal{M}=$ the nodes marked by the marking process. Provided $\ell_{n} \rightarrow \infty$ and $\ell_{n}<\sqrt{n / 3 \log n}$, the two quantities rarely differ, so we have the following corollary to Theorem 8 
Corollary $10 E\left(C_{k}^{\prime}\right) \geq E\left(C_{k}\right)-O\left(n^{2} e^{-n / \ell_{n}^{2}}\right)$.

Proof:

$$
E\left(C_{k}^{\prime}\right) \geq E\left(C_{k}^{\prime} \mid M=n\right) \mathbf{P}_{n}(M=n) .
$$

If $M=n$, i.e. if $\mathcal{M}=V$ and all nodes in the network are marked, then $\mathcal{C}_{k}(V)=\mathcal{C}_{k}(\mathcal{M})$. Therefore $E\left(C_{k}^{\prime} \mid M=n\right)=E\left(C_{k} \mid M=n\right)$, and

$$
E\left(C_{k}^{\prime} \mid M=n\right) \mathbf{P}(M=n)=E\left(C_{k}\right)-E\left(C_{k} \mid M \neq n\right) \mathbf{P}(M \neq n) .
$$

Combining (27) with (28), we get

$$
\begin{gathered}
E\left(C_{k}^{\prime}\right) \geq E\left(C_{k}\right)-E\left(C_{k} \mid M \neq n\right) \mathbf{P}(M \neq n) \\
\geq E\left(C_{k}\right)-n \mathbf{P}(M \neq n) \geq E\left(C_{k}\right)-O\left(n^{2} e^{-n / \ell_{n}^{2}}\right) .
\end{gathered}
$$

